\title{
Pemetrexed single agent chemotherapy in previously treated patients with locally advanced or metastatic non-small cell lung
}

\section{cancer}

\author{
Francesca Russo*1, Alessandra Bearz ${ }^{2}$, Gianni Pampaloni ${ }^{1}$ for the \\ investigators of the Italian Pemetrexed monotherapy of NSCLC group
}

\author{
Address: ${ }^{1}$ Medical Department, Eli Lilly Italia S.p.A. Sesto Fiorentino (FI), Italy and ${ }^{2}$ Oncologia Medica, Centro di Riferimento Oncologico Aviano \\ (PN), Italy \\ Email: Francesca Russo* - russo_francesca@lilly.com; Alessandra Bearz - abearz@cro.it; Gianni Pampaloni - pampaloni_gianni@lilly.com; the \\ investigators of the Italian Pemetrexed monotherapy of NSCLC group - russo_francesca@lilly.com \\ * Corresponding author
}

Published: 31 July 2008

BMC Cancer 2008, 8:216 doi:10.1186/147|-2407-8-216

This article is available from: http://www.biomedcentral.com/147I-2407/8/216

(c) 2008 Russo et al; licensee BioMed Central Ltd.

This is an Open Access article distributed under the terms of the Creative Commons Attribution License (http://creativecommons.org/licenses/by/2.0), which permits unrestricted use, distribution, and reproduction in any medium, provided the original work is properly cited.

\begin{abstract}
Background: The main objective of this study was to evaluate the safety of second-line pemetrexed in Stage IIIB or IV NSCLC.

Methods: Overall, 95 patients received pemetrexed $500 \mathrm{mg} / \mathrm{m}^{2}$ i.v. over Day I of a $2 \mathrm{I}$-day cycle. Patients also received oral dexamethasone, oral folic acid and i.m. vitamin BI2 supplementation to reduce toxicity. $\mathrm{NCI}$ CTC 2.0 was used to rate toxicity. All the adverse events were graded in terms of severity and relation to study treatment. Dose was reduced in case of toxicity and treatment was delayed for up to 42 days from Day I of any cycle to allow recovering from study drug-related toxicities. Tumor response was measured using the RECIST criteria.

Results: Patients received a median number of 4 cycles and $97.8 \%$ of the planned dose. Overall, 75 patients (78.9\% of treated) reported at least one adverse event: 34 (35.8\%) had grade 3 as worst grade and only $5(5.2 \%)$ had grade 4 . Drug-related events occurred in $57.9 \%$ of patients. Neutropenia (8.4\%) and leukopenia (6.3\%) were the most common grade $3 / 4$ hematological toxicities. Grade 3 anemia and thrombocytopenia were reported in $3.2 \%$ and $2.1 \%$ of patients, respectively. Diarrhea (6.3\%), fatigue (3.2\%) and dyspnea (3.2\%) were the most common grade $3 /$ 4 non-hematological toxicities. The most common drug-related toxicities (any grade) were pyrexia (II.6\%), vomiting, nausea, diarrhea and asthenia (9.5\%) and fatigue (8.4\%). Tumor Response Rate (CR/PR) in treated patients was $9.2 \%$. The survival at 4.5 months (median follow-up) was $79 \%$ and the median PFS was 3.1 months. Twenty patients $(21.1 \%)$ died mainly because of disease progression.
\end{abstract}

Conclusion: Patients with locally advanced or metastatic NSCLC could benefit from second-line pemetrexed, with a low incidence of hematological and non-hematological toxicities. 


\section{Background}

Lung cancer is more often diagnosed and is by far the most common cause of death from cancer in both genders worldwide $[1,2]$. Almost $80 \%$ of lung cancers can be classified as Non Small Cell Lung Cancer (NSCLC), with 65\% to $75 \%$ of cases presenting as locally advanced (Stage III) or metastatic disease (Stage IV) $[3,4]$.

Significant improvements in median survival in advanced NSCLC patients have been achieved with the use of platinum-based chemotherapy [5], particularly in patients with good performance status, and with newer cytotoxic agents, such as gemcitabine, paclitaxel, docetaxel or vinorelbine [6,7]. It is actually believed that the next significant advance in the treatment of NSCLC might derive from the use of targeted agents, as monotherapy or in combination with standard chemotherapy regimens, without increasing toxicity [6].

Pemetrexed is a new multi-target antifolate agent approved for the treatment of malignant pleural mesothelioma and NSCLC. Pemetrexed exerts its cytotoxic effect through inhibition of Thymidylate Synthase, Dihydrofolate Reductase and Glycinamide Ribonucleotide Formyl Transferase [8], which are involved in DNA synthesis and folate metabolism [9]. The multiple inhibitions of several key folate-requiring enzymes may account both for the antitumor activity and the potential cytotoxic effect of pemetrexed. It has been found that the hematological and non-hematological toxicities of pemetrexed can be reduced through routine vitamin supplementation (folic acid and vitamin B12), without loss of efficacy [10].

Several reports have documented the effects of pemetrexed given as a single agent and in combination in firstor second-line chemotherapy in advanced NSCLC [11]. In phase II trials, pemetrexed has shown high efficacy and favorable toxicity when given in combination with platinum agents, gemcitabine and vinorelbine [12,13]. A recent phase III trial that compared pemetrexed with docetaxel in previously treated NSCLC patients showed equivalent efficacy in response rate and survival, and significantly less toxicity in the pemetrexed group when compared to docetaxel [14].

According to Italian legislation, which establishes a drug dispensing as 'therapeutic use' prior to approval for use in local market, this study was aimed at extending the clinical experience with pemetrexed in pretreated patients with locally advanced or metastatic NSCLC.

\section{Methods}

Patients

Adult patients of both genders with locally advanced or metastatic NSCLC (Stage IIIB or IV at entry), previously treated with no more than two chemotherapy regimens for advanced disease, were eligible for the study. Prior chemotherapy and/or radiotherapy (excluding pemetrexed) were to be completed at least 2 weeks prior to study enrollment and the patients should have recovered from any acute toxic effect of previous therapy. Prior radiation therapy allowed to $<25 \%$ of the bone marrow. Moreover, eligible patients were required to have a ECOG Performance Status 0 to 2, an estimated life expectation of at least 8 weeks, and an adequate bone marrow reserve. Patients with evidence of hepatic or renal insufficiency, active infection, inability to take folic acid, vitamin B12 supplementation or corticosteroids, signs of malnourishment or $>10 \%$ weight loss in the past 6 weeks, or others serious concomitant disorders (including oncologic emergencies) were excluded from the study. Pregnant or breastfeeding females were also not allowed to taking part in the study, as well as an adequate contraceptive method was to be used for the whole study duration. Patients were to be discontinued from the study in the case of evidence of progressive disease or unacceptable toxicity despite dose adjustment.

The participant patients gave their written informed consent prior to enter in the study. The study protocol and the informed consent form were reviewed and approved by the Independent Ethics Committees of each participating center prior to any study-related procedure was started.

\section{Treatments}

Pemetrexed $500 \mathrm{mg} / \mathrm{m}^{2}$ (Alimta ${ }^{\circledR}$, Eli Lilly and Company, Indianapolis, IN) was administered i.v. over approximately 10 minutes on Day 1 of a 21-day cycle. Dexamethasone $4 \mathrm{mg}$ or equivalent corticosteroid was taken orally twice daily on the day before, the day of, and the day after each dose of pemetrexed. Folic acid supplementation 350 to $600 \mu \mathrm{g}$ or equivalent was taken orally daily beginning approximately 1 to 2 weeks prior to the first dose of pemetrexed and continued until 3 weeks after study therapy discontinuation. Patients also received a $1000 \mu \mathrm{g}$ vitamin B12 i.m. injection approximately 1 to 2 weeks prior to the first dose of pemetrexed, to be repeated approximately every 9 weeks until 3 weeks after study therapy discontinuation.

Any patient who required a pemetrexed dose reduction due to hematological or non-hematological toxicities was treated further according to dose reductions. Any patient requiring $>2$ reductions due to toxicity was to be withdrawn from study therapy. Treatment could have been delayed for up to 42 days from Day 1 of any cycle to allow recovering from study drug-related toxicities.

No other chemotherapy, immunotherapy, hormonal cancer therapy, radiation therapy, surgery for cancer, or any 
other experimental medications was permitted during the study. Disease progression requiring alternative antitumor treatment led to early discontinuation of study therapy. If patient required radiotherapy treatment (both palliative or not) during the study, pemetrexed was discontinued until 2 weeks after the completion of radiation treatment.

The use of growth factors was not allowed by study protocol.

\section{Outcome measures}

The analysis of safety was the primary endpoint of the study. The safety measures used in the study included adverse events, physical examinations and clinical laboratory tests (hematology, blood chemistry and urinary creatinine clearance). All the adverse events were evaluated in terms of severity and relation to study treatment, while toxicities according to the National Cancer Institute Common Toxicity Criteria (NCI CTC) version 2.0 [15].

The evaluation of the best tumor response rate was performed at the end of the treatment period and the Response Evaluation Criteria in Solid Tumors (RECIST) were recommended [16]. The progression free survival (PFS) was the time from study entry to disease progression or death, while the overall survival time was defined as the time from study entry to death due to any cause. Investigators followed-up the survival status of patients who had discontinued study therapy.

Adverse events were considered those emerging during treatment or present at baseline and worsening during the study.

\section{Statistics}

The statistical analysis was performed using the SAS version 8.2 (Cary, NC, US). The analyses were mainly descriptive: summary statistics were given for patient characteristics, treatment administration and all safety variables (laboratory tests and adverse events). Adverse events were coded using the MedDRA dictionary. Tumor Response Rate (complete response/partial response [CR/ $\mathrm{PR}]$ ) was calculated considering all patients who received at least one dose of study drug. PFS and overall survival time were analyzed by means of Kaplan-Meier method.

\section{Results}

A total of 102 patients were enrolled in 35 Italian centers from December 2004 to May 2005. The demographic and baseline clinical condition of treated patients are summarized in Table 1. Most of the patients were in good Performance Status: 87 patients (93.6\% of valuable patients) were ECOG PS 0 to 1 . Adenocarcinoma/Neoplasia NOS
Table I: Demographic and baseline clinical condition of the treated patients $(n=95)$

\begin{tabular}{lc}
\hline Age, years: mean \pm SD (range) & $62.4 \pm 10.6(25-82)$ \\
Age ranges, N (\%): & $11(11.6)$ \\
$\leq 50$ & $21(22.1)$ \\
$51-60$ & $44(46.3)$ \\
$6 I-70$ & $19(20.0)$ \\
$>70$ & \\
\hline Sex: N (\%) & $72(75.8)$ \\
Males & $23(24.2)$ \\
Females & \\
\hline Weight, kg: mean \pm SD (range) & \\
\hline NSCLC, histological type: N (\%) & $13.6(41-110)$ \\
Neoplasia NOS, adenocarcinoma & $46(48.4)$ \\
Squamous cells & $26(27.4)$ \\
Large cells & $2(2.1)$ \\
Other & $21(22.1)$ \\
\hline ECOG, score: N (\%) & \\
0 & $58(62.4)$ \\
I & $29(31.2)$ \\
2 & $5(5.4)$ \\
3 & $1(1.1)$ \\
Not available & 2 \\
\hline
\end{tabular}

$\mathrm{N}=$ number of patients, \% refers to total of treated patients with available data

was the most frequent histological type, representing approximately half of cases.

Ninety-five of them (93.1\% of enrolled) received at least one dose of study drug. Seven patients were included but did not receive study drug ( 2 because of physician decision, 2 patient decision, 2 deaths, 1 entry criteria violation).

The median received cycles was 4.0 (range 1-15), while the median number of weeks of treatment was 12.1 (range 1.4-57.3). Fifty patients (52.6\%) had dose modification at least in one cycle: pemetrexed dose was reduced due to adverse events in 12 patients and was delayed (mostly due to adverse events or conflict in scheduling) in 48 patients. The median relative dose intensity was $97.8 \%$ (range 63.1-104.0). Deviations from the scheduled dosing of dexamethasone, folic acid and vitamin B12 were reported in 3, 7 and 8 patients, respectively.

The main reasons for treatment discontinuation were lack of efficacy (46 patients, 48.4\%), physician decision (13, $13.7 \%)$, objective responses $(13,13.7 \%)$ and patient decision $(8,8.4 \%)$. Fifteen patients had protocol violation and the most common was the incorrect dose reduction due to toxicity (7 patients). 


\section{Safety}

Seventy-five patients (78.9\% of treated) reported at least one adverse event during the study, 34 patients (35.8\%) and 5 patients $(5.2 \%)$ experienced grade 3 and grade 4 adverse events, respectively. Fifty-five patients (57.9\%) had adverse events considered by physicians as possibly related to study treatment.

Table 2 shows adverse events reported in $\geq 5 \%$ of patients by preferred term and study drug relationship. The most common adverse events were pyrexia (reported in $26.3 \%$ of treated patients and judged as drug-related in $11.6 \%$ ), asthenia (overall $13.7 \%$ of patients, drug-related in 9.5\%) and dyspnea (overall $11.6 \%$ of patients, drug-related in only one case). General disorders and administration site conditions (26.3\%), gastrointestinal disorders $(23.2 \%)$ and blood and lymphatic system disorders (22.1\%) were the system organ classes with the highest incidence of adverse events related to pemetrexed.

The highest incidences of CTC grade 3/4 adverse events were reported as blood and lymphatic system disorders (17.9\%), gastrointestinal disorders (9.5\%) and general disorders and administration site conditions (9.5\%). Grade 3 adverse events reported in > 1 patient included anemia (3 patients), leukopenia (6), neutropenia (6), thrombocytopenia (2), diarrhea (6), nausea (2), vomiting (2), fatigue (3), mucosal inflammation (2), thrombocytopenia (2), and dyspnea (3 patients). Grade 4 adverse events included neutropenia ( 2 patients), and acute myo-

Table 2: Treatment-emergent adverse events reported by $\geq 5 \%$ of treated patients by preferred term and study drug relationship: data are number of patients with rates in brackets ( $N=95)$

\begin{tabular}{lcc}
\hline & All Causalities & Treatment Related \\
\hline Patients with $\geq$ I adverse event & $75(78.9)$ & $55(57.9)$ \\
\hline Pyrexia & $25(26.3)$ & $11(11.6)$ \\
Asthenia & $13(13.7)$ & $9(9.5)$ \\
Dyspnea & $11(11.6)$ & $1(1.1)$ \\
Neutropenia & $10(10.5)$ & $10(10.5)$ \\
Vomiting & $10(10.5)$ & $9(9.5)$ \\
Diarrhea & $10(10.5)$ & $9(9.5)$ \\
Anemia & $10(10.5)$ & $8(8.4)$ \\
Nausea & $9(9.5)$ & $9(9.5)$ \\
Fatigue & $9(9.5)$ & $8(8.4)$ \\
Cough & $8(8.4)$ & $3(3.2)$ \\
Anorexia & $6(6.3)$ & $1(1.1)$ \\
Leucopenia & $5(5.3)$ & $5(5.3)$ \\
Mucosal inflammation & $5(5.3)$ & $5(5.3)$ \\
Thrombocytopenia & $5(5.3)$ & $5(5.3)$ \\
Rash & $5(5.3)$ & $4(4.2)$ \\
Chest pain & $5(5.3)$ & $1(1.1)$ \\
Peripheral Edema & $5(5.3)$ & $1(1.1)$ \\
& & \\
\hline
\end{tabular}

cardial infarction, myocardial ischemia and melaena (all occurred in the same patient).

A total of 20 patients (21.1\% of treated population) had at least one event fulfilling the criteria for a serious adverse event; 5 of them were considered drug-related (neutropenia in 2 patients, diarrhea, pyrexia, melaena, anemia and vomiting in 1$)$. Overall, 19 patients $(20.0 \%)$ died due to disease progression: 5 patients $(5.3 \%)$ died while on treatment or within 30 days of treatment discontinuation, 14 died after 30 days from treatment discontinuation. One patient died due to cardiac failure.

Hematological assessments were performed on 90 out the 95 treated patients. Table 3 shows the out of range hematological values observed during treatment (NCIC-CTC grading). NCIC-CTC grade 3 hematological toxicities were the following: anemia $2.2 \%$ of patients, leucopenia $17.8 \%$, neutropenia $18.9 \%$, and thrombocytopenia $4.4 \%$.

No clinically relevant changes in vital signs were reported during the study.

\section{Efficacy}

Table 4 shows the results of the overall tumor best response in the treated patients population with measurable disease at baseline $(\mathrm{N}=87)$ : 8 patients $(9.2 \% ; 95 \%$ : 4.1 to 17.3 ) were responders ( 1 CR and 7 PR), 23 patients (26.4\%) were stable on their disease, 49 patients (56.3\%) had disease progression as best response and 7 patients $(8.0 \%)$ were not evaluable for response.

The Kaplan-Meier survival analysis at 4.5 months (median follow-up) was $79 \%$ (95\% CI: 71 to $88 \%$ ). The median PFS was 3.1 months (95\% C.I. 2.4 to 3.8 ).

\section{Discussion}

Previous phase 2 studies have indicated that pemetrexed (Alimta ${ }^{\circledR}$ ) has clinical activity in NSCLC. A comparative trial of Pemetrexed and docetaxel (Eli Lilly Protocol H3EMC-JMEI), compared 571 patients with locally advanced or metastatic NSCLC who had previously been treated with chemotherapy.

Table 3: Hematology abnormalities observed during treatment (worst NCIC-CTC grading): data are number of patients with rates in brackets $(\mathbf{N}=\mathbf{9 0})$

\begin{tabular}{lcc}
\hline Laboratory Parameter & Any grade $\geq$ I & Grades 3-4 \\
\hline Hemoglobin & $63(70.0)$ & $2(2.2)$ \\
Neutrophils & $50(55.6)$ & $17(18.9)$ \\
Platelets & $37(41.1)$ & $4(4.4)$ \\
WBCs & $57(63.3)$ & $16(17.8)$
\end{tabular}


Table 4: Results of the overall tumor response in the treated population: data are number of patients with rates in brackets

\begin{tabular}{lc}
\hline Best Overall Tumor Response & $\begin{array}{c}\text { Treated Population } \\
(\mathrm{N}=87)^{*}\end{array}$ \\
\hline Complete Response (CR) & $\mathrm{I}(\mathrm{I} . \mathrm{I})$ \\
Partial Response PR) & $7(8.0)$ \\
Response Rate (CR + PR) & $8(9.2)$ \\
Stable Disease/No Response (SD) & $23(26.4)$ \\
Progressive Disease (PD) & $49(56.3)$ \\
Not Evaluable & $7(8.0)$
\end{tabular}

*Numbers and rates refer to the amount of patients assessed for tumor response (response was not available in 8 patients in the treated population)

The primary objective of this study was to confirm the safety profile of pemetrexed $\left(500 \mathrm{mg} / \mathrm{m}^{2}\right.$ dose, day 1 of a 21-day cycle) as second line treatment in patients with locally advanced or metastatic (Stage IIIB or IV) NSCLC. Pemetrexed was supplemented with dexamethasone, folic acid and vitamin B12 was given every 21 days. This regimen is recommended based on previous experiences $[17,14]$, which showed a significant improved tolerance when pemetrexed is given with corticosteroids and vitamins supplementation.

The secondary objective of the study was to assess the response rate in patients with measurable disease according to the RECIST criteria.

In this study 95 patients were examined. The majority of patients (>90\%) had good clinical conditions (ECOG PS 0 or 1 ). The median number of cycles received was 4 and the median number of weeks of treatment was 12.1. Pemetrexed was well tolerated. The safety profile of pemetrexed did not differ from what observed in previous phase I/II studies and in the large phase III study comparing pemetrexed and docetaxel as second-line treatment in locally advanced or metastatic NSCLC [14]. In the latter trial, which led to the regulatory approval of pemetrexed as monotherapy for the second-line treatment of NSCLC, the incidence of hematological toxicities (e.g. grade $3 / 4$ neutropenia, febrile neutropenia, and neutropenia with infections) and other drug-related adverse events was significantly lower with pemetrexed than with docetaxel. The results of the present study confirm the favorable toxicity profile of pemetrexed when given over $500 \mathrm{mg} / \mathrm{m}^{2}$ and supplemented by vitamin B12 and folic acid.

Vitamin supplementation significantly reduces the incidence of grade 3-4 hematological toxicity, as shown in a previous trial comparing pemetrexed administered with or without vitamins [18].
The most frequent hematological toxicities were neutropenia and anemia (any grade) and the most frequent nonhematological toxicities were pyrexia, fatigue and dyspnea (any grade).

In the population of patients with measurable disease at baseline the observed response rate was $9.2 \%$ and it was similar to the Response Rate reported in the randomized phase III study ( $8.8 \%$ ) when pemetrexed was compared to docetaxel [14].

It is generally agreed that response rate cannot be taken as indicator of clinical benefit in pretreated patients with locally advanced or metastatic NSCLC and the relationship between response rate and improved survival is unclear, so that response rate cannot be considered as a surrogate endpoint. However, a prolonged survival in pretreated advanced NSCLC patients has been observed, in spite of a response rate lower than $10 \%$. This therefore suggests a possible contribution from cytotoxic agents to disease stabilization and to the clinical benefit observed [19].

In our study, the survival at approximately 4 months (median follow-up time) was $79 \%$ and the median progression-free survival was 3.1 months, what is in line with the reported survival rate in the reported phase III trial by Hanna et al. in the comparative study vs Docetaxel [14].

It is well known that, especially in $2^{\text {nd }}$ line, tolerability and toxicity profile of a cytotoxic combination might influence the choice of treatment, even when the efficacy parameters of possible therapies (e.g. survival, progression free survival and response rate) are similar. The duration of infusion, schedule administration and patients acceptance should be also taken into consideration for the choice of a regimen. When compared to the other agents currently approved for $2^{\text {nd }}$ line treatment in NSCLC, the 10-minutes infusion time of pemetrexed over Day 1 of a 21-day cycle might increase the convenience of the treatment and patient compliance.

Therefore, the study confirmed that pretreated patients with locally advanced or metastatic NSCLC will likely benefit from single-agent pemetrexed treatment (with vitamin supplementation), with an additional advantage in decreasing hematological (including febrile neutropenia) and non-hematological toxicities.

Since, due to a minor flaw in the original study design, there are no available data on whether patients were treated with pemetrexed in $2^{\text {nd }}$ or $3^{\text {rd }}$ line, it is not possible to assess any correlation between the number of previous lines of treatment and response to pemetrexed. 
It has been recently pointed out that the administration of Pemetrexed in combination with other agents (eg. Cisplatin, Carboplatin or gemcitabine) in the treatment of advanced NSCLC may provide further clinical benefits caused by its particular mode of action when blocking intracellular three enzymes system. A deeper knowledge about those enzyme system (eg: TS) may be used in future to identify patients responders to pemetrexed [20]. The use of targeted compounds to specific molecular pathways, given in addition to standard chemotherapy regimens, might represent the next step in the treatment of NSCLC and overall characteristics of pemetrexed makes it a candidate in a tailored therapies context.

The present study contributes to provide even more information on clinical experience with pemetrexed and further prospective randomized clinical trials will confirm pemetrexed (single agent or in combination) as a valid option for pretreated locally advanced or metastatic NSCLC patients.

\section{Competing interests}

The study was fully sponsored by Eli Lilly Italia. Francesca Russo and Gianni Pampaloni are employed at Eli Lilly Italia.

\section{Authors' contributions}

All authors have given substantial contributions to conception and design the study. FR and GP have given substantial contributions to analysis and interpretation of data, and in the revision of the manuscript. $A B$ has given relevant contributions in the recruitment of patients, in the critical revision of the manuscript, and in final approval prior to publication. All authors read and approved the final manuscript.

\section{Acknowledgements}

The authors thank Luca Cantini for his contribution in the medical writing of this manuscript and Sara Ballasio and Farma Resa for their technicaladministrative support. A special thank to Marco Pacini (Eli Lilly Italia), Giulia Calamai (Eli Lilly Italia) and Andrea Rossi (Eli Lilly Italia - member of European Medical Writers Association), for their supervision of the project and Marta Zanus (CROS Italia) for her contribution in performing statistical analysis.

The investigators of the Italian Pemetrexed monotheerapy of NSCLC group are:

Umberto Tirelli: Dipartimento oncologia Medica, C.R.O. (Centro di Riferimento Oncologico) Istituto Nazionale Tumori Aviano (PN); Roberto Bordonaro: Divisione di Oncologia Medica - Day Hospital, Azienda Ospedaliera Vittorio Emanuele II Catania; Claudio Verusio: Oncologia Medica, Ospedale di Circolo di Busto Arsizio - P.O. di Saronno (VA); Alberto Rosa Bian: Unità Operativa di Oncologia Medica, Ospedale Boldrini Thiene (VI); Maurizio Marangolo: Divisione di Oncologia Medica, Ospedale "S. Maria delle Croci" Ravenna; Angelo Raffaele Bianco: Dipartimento di Endocrinologia ed Oncologia Molecolare e Clinica,"Università Studi di Napoli Federico II - II Facoltà di Medicina e Chirurgia Policlinico Federico II"
Napoli; Francesco Grossi: Oncologia Medica A - $5^{\circ}$ piano, Istituto Nazionale Tumori Genova; Angelo Gambi: Centro Oncologico, Ospedale degli Infermi Faenza (FO); Guido Francini: U.O. Oncologia Medica, Pol. Le Scotte, Università degli Studi di Siena; Dr. Giorgio Sogno: Osp. San. Paolo di Savona; Paolo Manente: U.O. di Oncologia Medica, Unità Socio Sanitaria 8 Stabilimento Ospedaliero Castelfranco Veneto (TV); Nicola Gebbia: Servizio di Chemioterapia Antiblastica, Policlinico Universitario Paolo Giaccone Palermo; Fabrizio Artioli: Unità Operativa di Medicina Oncologica, Ospedale Civile S. Giacomo Carpi (MO); Andrea Ardizzoni Oncologia Medica, Az. Ospedaliera di Parma; Alessandro Masotti: Pneumologia, Ospedale Maggiore B. Trento di Verona; Ernesto Pozzi: Clinica di Malattie Apparato respiratorio, IRCCS Policlinico San Matteo Pavia; Salvatore Tumolo: U.O. Oncologia Medica, Osp. Santa Maria degli Angeli Pordenone; Rodolfo Passalacqua: Oncologia Medica, Azienda Ospedaliera Istituti Ospetalieri di Cremona; Clelia Casartelli: Struttura Semplice di Oncoematologia U.O. di Medicina, Ospedale "Valduce" Como; Franco Montanari: Azienda Ospedaliera di Vimercate Presidio Ospedaliero di Desio (MI); Giuseppe Colucci: Divisione di Oncologia Medica e Sperimentale, IRCCS Ospedale Oncologico Bari; Santi Barbera: U.O. di Pneumologia Oncologica, Presidio Ospedaliero Mariano Santo - Azienda Ospedaliera di Cosenza Cosenza; Sergio Ricci: U.O. Oncologia Medica, Ospedale Santa Chiara Pisa; Lucio Trodella: Radioterapia, Università Cattolica Sacro Cuore, Policlinico "A. Gemelli" Roma; Vincenzo Valentini: Radioterapia, Università Cattolica Sacro Cuore, Policlinico "A. Gemelli" Roma; Giovanni Mantovani: Oncologia Medica I, Policlinico Universitario Monserrato, Monserrato (CA); Alberto Ravaioli: Oncologia Medica, Ospedale Civile degli Infermi Rimini; Silvio Monfardini: Oncologia Medica, Azienda Ospedaliera di Padova; Sandro Barni: Oncologia Medica, Ospedali Riuniti di Treviglio e Caravaggio Treviglio (BG); Raffaella Felletti: Divisione di pneumologia - Dipartimento di Medicina Specialistica, Ospedale San Martino - Padiglione Maragliano Genova; Franco Testore: "U.O.A. Oncologia", Ospedale Civile di Asti; Massimo Aglietta - Divisione di Oncologia Medica ed Ematologia, IRCC- Isutituto per la Ricerca e la Cura del Cancro Candiolo (TO); Antonio Ardizzoia: Ospedale S.Gerardo Monza (MI); Roberto Bollina: U.O.Oncologia MedicaF.B.F, Ospedale San Giuseppe Milano; Giorgio Cruciani: Servizio di Oncologia, Ospedale Umbero I di Lugo (Az. USL di Ravenna) Lugo di Romagna (Ra); Enzo Pasquini: Reparto di Oncologia Medica, Ospedale Cervesi Cattolica (RN).

\section{References}

I. Parkin DM, Bray F, Ferlay J, Pisani P: Global cancer statistics, 2002. CA Cancer J Clin 2005, 55(2):74-I08.

2. Ho C, Davies AM, Lara PN Jr, Gandara DR: Second-line treatment for advanced-stage non-small-cell lung cancer: current and future options. Clin Lung Cancer 2006, 7(Suppl 4):SI I8-25.

3. Wakelee $\mathrm{H}$, Belani CP: Optimizing first-line treatment options for patients with advanced NSCLC. Oncologist 2005, I O(Suppl 3): $1-10$.

4. Walling J: Chemotherapy for advanced non-small-cell lung cancer. Resp Med 1994, 88:649-657.

5. Molina JR, Adjei AA, Jett JR: Advances in chemotherapy of nonsmall cell lung cancer. Chest 2006, I30(4): 1211 -9.

6. Blackhall FH, Shepherd FA, Albain KS: Improving survival and reducing toxicity with chemotherapy in advanced non-small cell lung cancer: a realistic goal? Treat Respir Med 2005, 4(2):71-84.

7. Sorenson S, Glimelius B, Nygren P: A systematic overview of chemotherapy effects in non-small cell lung cancer. Acta Oncol 200I, 40(2-3):327-39.

8. Shih C, Chen VJ, Gossett LS, Gates SB, MacKellar WC, Habeck LL, Shackelford KA, Mendelsohn LG, Soose DJ, Patel VF, Andis SL, Bewley JR, Rayl EA, Moroson BA, Beardsley GP, Kohler W, Ratnam M, Schultz RM: LY23 a pyrrolo[2,3-d]pyrimidine-based antifolate that inhibits multiple folate-requiring enzymes. Cancer Res 1514, 57(6): $1|16-1| 23$. 
9. Schilsky RL: Antimetabolites. In The Chemotherapy Source Book Edited by: Perry MC. Baltimore (MD): Williams \& Wilkins; 1992:30I-315.

10. Solomon B, Bunn PA Jr: Clinical activity of pemetrexed: a multitargeted antifolate anticancer agent. Future Oncol 2005, I(6): $733-46$.

II. Hanauske AR, Dittrich C, Otero J: Overview of phase I/II pemetrexed studies. Oncology (Williston Park) 2004, I8(13 Suppl 8): $18-25$.

12. Scagliotti GV: Pemetrexed plus carboplatin or oxaliplatin in advanced non-small cell lung cancer. Semin Oncol 2005, 32(2 Suppl 2):S5-8.

13. Manegold C: Pemetrexed: its promise in treating non-smallcell lung cancer. Oncology (Williston Park) 2004, I8(8 Suppl 5):43-8.

14. Hanna N, Shepherd FA, Fossella FV, Pereira JR, De Marinis F, von Pawel J, Gatzemeier U, Tsao TC, Pless M, Muller T, Lim HL, Desch C, Szondy K, Gervais R, Shaharyar, Manegold C, Paul S, Paoletti P, Einhorn L, Bunn PA Jr: Randomized phase III trial of pemetrexed versus docetaxel in patients with non-small-cell lung cancer previously treated with chemotherapy. J Clin Oncol 2004, 22(9): 1589-97.

15. Cancer Therapy Evaluation Program: Common Toxicity Criteria, Version 2.0. DCTD, NCI, NIH, DHHS 1998.

16. Therasse P, Arbuck SG, Eisenhauer EA, Wanders J, Kaplan RS, Rubinstein L, Verweij J, Van Glabbeke M, Van Oosterom AT, Christian MC, Gwyther SG: New guidelines to evaluate the response to treatment in solid tumors. European Organization for Research and Treatment of Cancer, National Cancer Institute of the United States, National Cancer Institute of Canada. I Natl Cancer Inst 2000, 92(3):205- 16.

17. Smit EF, Mattson K, von Pawel J, Manegold C, Clarke S, Postmus PE: ALIMTA (pemetrexed disodium) as second-line treatment of non-small-cell lung cancer: a phase II study. Ann Oncol 2003, I4(3):455-60.

18. Scagliotti GV, Shin DM, Kindler HL, Vasconcelles MJ, Keppler U, Manegold C, Burris H, Gatzemeier U, Blatter J, Symanowski JT, Rusthoven J]: Phase II study of pemetrexed with and without folic acid and vitamin B I 2 as front-line therapy in malignant pleural mesothelioma. J Clin Oncol 2003, 2 I (8): I556-6I.

19. Ramalingam $S$, Sandler $A B$ : Salvage therapy for advanced nonsmall cell lung cancer: factors influencing treatment selection. Oncologist 2006, I I (6):655-65.

20. Monnerat C, Le Chevalier T: Review of the pemetrexed and gemcitabine combination in patients with advanced-stage non-small cell lung cancer. Ann Oncol 2006, I7(Suppl 5):86-90.

\section{Pre-publication history}

The pre-publication history for this paper can be accessed here:

http://www.biomedcentral.com/1471-2407/8/216/pre pub

Publish with Biomed Central and every scientist can read your work free of charge

"BioMed Central will be the most significant development for disseminating the results of biomedical research in our lifetime. "

Sir Paul Nurse, Cancer Research UK

Your research papers will be:

- available free of charge to the entire biomedical community

- peer reviewed and published immediately upon acceptance

- cited in PubMed and archived on PubMed Central

- yours - you keep the copyright

Submit your manuscript here:

http://www.biomedcentral.com/info/publishing_adv.asp
BioMedcentral 